\title{
Material Characterisation of Nanowires with Intrinsic Stress.
}

\author{
Shaun Mills ${ }^{\dagger}$, John E. Sader ${ }^{\perp}$, John J. Boland ${ }^{\dagger}$ \\ ${ }^{\dagger}$ School of Chemistry, Trinity College Dublin, Dublin 2, Ireland \\ $\S$ Centre for Research on Adaptive Nanostructures and Nanodevices (CRANN), Trinity \\ College Dublin, Dublin 2, Ireland \\ ${ }^{\perp}$ School of Mathematics and Statistics, University of Melbourne, Victoria 3010, Australia
} KEYWORDS

Residual stress, doubly-clamped, nanowires, Young's modulus, Euler-Bernoulli 


\section{ABSTRACT}

When fabricating nanowires in a doubly-clamped beam configuration it is possible for a residual axial stress to be generated. Here, we show that material characterisation of metal and semiconductor nanowires subjected to residual axial stress can be problematic. Benchmark measurements of the Young's modulus of nanowires are performed by sectioning a doublyclamped nanowire into two cantilevered wires, eliminating residual axial stress. Use of models for doubly-clamped beams that incorporate the effects of residual stress are found to lead to ambiguity in the extracted Young's modulus as a function of displacement fit range, even for nanowires with no residual stress. This is due to coupling of bending and axial stress effects at small displacements, and the limited displacement range of force curves prior to fracture or plastic deformation. This study highlights the importance of fabricating metal and semiconductor nanowires that exhibit little or no residual axial stress for materials characterisation.

\section{TEXT}

Nanowires (NWs) have attracted considerable interest for their potential use as the active components of mechanical and electromechanical devices. In order for nanowires to be incorporated into devices it is imperative that their mechanical properties are fully understood. The characterisation of nanomechanical properties is challenging in the first instance simply due to the required handling and controlled mechanical manipulation that this involves. Secondly, the small sizes and large surface areas make nanomaterials susceptible to external environmental factors that may influence measured properties. For example, the interaction with a supporting substrate may lead to the incorporation of residual stresses into these materials. If these stresses are not accounted for in the mechanical characterisation of these wires, there is significant potential to report erroneous results. It is well established that 
mechanical properties of nanowires can be vastly different to that of their bulk counterparts $\mathrm{s}^{1,2,3,4}$ therefore, great care is required in the recording and analysis of nanomechanical measurements.

Different techniques have been employed to measure the mechanical properties of nanomaterials. Electron microscopy based techniques have been used to examine the resonance response of piezomechanically driven 1 -d nanowire materials ${ }^{5,6}$ in addition to in-situ tensile testing using a force measurement strain stage. ${ }^{7,8}$ Atomic Force Microscopy (AFM) based techniques include nanoindentation methods ${ }^{9,10}$ and the exploitation of both normal ${ }^{11}$ and lateral $^{12,1,13}$ deflection to measure the mechanical response of nanowires. The AFM approach has the advantage that it can be used to simultaneously measure the mechanical and electrical properties of nanowires, which in turn enables determination of the material's Poisson's ratio. ${ }^{14}$

AFM nanomechanical measurements are typically performed in a doubly-clamped freelysuspended-beam configuration. Accordingly, it is important that the adhesion between the nanowire and the substrate is sufficient to provide mechanical clamping. In instances where the natural adhesion is weak it is necessary to mechanically clamp the wires to define a pinning length, which can be accomplished using EBL patterned metal contacts or by local FIB-based metal deposition. The free-suspension of the nanowire between the clamps is insured by placing the pinned length of the wire over a hole ${ }^{15}$ or a trench in the substrate or by conducting the experiment in a liquid medium. ${ }^{16}$ For accurate mechanical measurements, it is important to know or account for the intrinsic (residual) stress state of the material. This is particularly important for nanomaterials since surface adhesion and/or clamping which are necessary for mechanical measurement may also potentially impact the mechanical response of the material itself.

Here we show that in many instances the deposition and clamping techniques currently in use can lead to the incorporation of unintended residual stresses. We demonstrate this by 
performing mechanical measurements on doubly-clamped wires and show that the mechanical response cannot be accounted for by the standard model developed previously for nanowires by Heidelberg et al. ${ }^{12}$ In the presence of residual stress, the force-displacement $(F-d)$ behaviour can be poorly described by the model preventing the determination of the materials mechanical properties. We demonstrate that residual stress can be naturally present in such wires. This is accomplished by using a focussed ion beam to section the suspended wire into two singleclamped cantilevers, each of which are well described by conventional Euler-Bernoulli beam mechanics and yield Young's moduli that are very close to the expected bulk values.

We begin by describing the ideal (zero-residual tension) doubly-clamped free-suspended wire configuration first considered by Heidelberg et al., ${ }^{12}$

$$
F_{\text {center }}=\frac{192 E I}{L^{3}} f(\alpha) \Delta z_{\text {center }}
$$

where $F_{c e n t e r}$ is the force applied perpendicular to the NW axis at its central point, $E$ is the Young's modulus of the wire, $I$ is the areal moment of inertia for a cylindrical wire and $L$ is the pinned length. The function $f(\alpha)$ is defined

$$
f(\alpha)=\frac{\alpha}{48-\frac{192 \tanh (\sqrt{\alpha} / 4)}{\sqrt{\alpha}}}
$$

where $\alpha$ is related to the displacement $\Delta z_{\text {center }}$ of the wire by

$$
\alpha=\frac{6 \epsilon(140+\epsilon)}{350+3 \epsilon}, \quad \epsilon=\left(\frac{2 \Delta \mathrm{z}_{\text {center }}}{\mathrm{R}}\right)^{2},
$$

This model assumes the wire is not subjected to a residual axial stress. The $f(\alpha)$ function may be approximated so that the force $F_{\text {centre }}$ comprises two terms: (i) a bending term that is linear in the displacement $\Delta \mathrm{Z}_{\text {center }}$ and (ii) a tensile term that is cubic in the displacement $\Delta \mathrm{Z}_{\text {center. }}{ }^{12} \mathrm{We}$ note that residual stress in the wire will enhance the contribution of the first term but not the 
second. As such, it will affect the transition from the linear to the cubic dependence on $\Delta z_{\text {center }}$ in Eq. (1).

To test for the presence of residual stress we repeat the experiment outlined by Heidelberg et $a l .{ }^{12}$ to determine if the $F-d$ response of our wires fits the model described in Eq. (1). Silver nanowires (AgNWs) pre-dispersed in isopropanol are dropcast onto a $\mathrm{SiO}_{2}$ surface with prefabricated trenches. Due to the volatile nature of the solvent it evaporates leaving the AgNWs deposited on the surface. Wires that are suspended perpendicularly over trenches are identified for mechanical measurements. Trenches are $250 \mathrm{~nm}$ deep and have widths of approximately 2 $\mu \mathrm{m}$, giving a ratio of trench width to nanowire diameter of $\sim 30$ times. This is the optimal ratio for these measurements because it eliminates wire droop due to gravity and minimises the role of the clamps during the doubly-clamped beam AFM measurements. ${ }^{2}$ In order to define the pinning length, $L$, the nanowires are pinned using Pt-electron beam induced metal deposition as shown in the inset of Figure 1b. The pinning points are deposited as close to the trench edges as possible to eliminate wire/substrate friction affects during the doubly-clamped beam AFM measurements.

For the doubly-clamped beam experiments $75 \mathrm{kHz}$ Budget Sensor AFM cantilevers with nominal spring constants of $3 \mathrm{~N} / \mathrm{m}$ are used. The calibration procedure for these cantilevers is described elsewhere. ${ }^{17}$ An Asylum MFP-3D AFM equipped with lateral lithography suite and closed $x-y$ loop is used to elastically load the NWs in ambient conditions at room temperature. Prior to manipulation the NW long axis is aligned parallel to the cantilever axis to avoid slippage between the NW and AFM cantilever tip during mechanical deformation. Figure 1a shows the loading/unloading behaviour of an individual AgNW. Prior to loading there is no lateral force experienced by the tip indicating that the tip is not in contact with the bottom of the trench. The tip then loads the wire resulting in an increasing force response. At a displacement of approximately $100 \mathrm{~nm}$ the tip reverses direction and unloads the wire. The tip 
then separates from the wire where the force on the tip returns to its preload value. This is repeated twice more with each loading curve being the same as the one previous as can be observed in Figure 1a. Additional mechanical properties such as yield point and yield strength can be obtained ${ }^{2,18,17}$ by plastically deforming the wire, however this is not the purpose of the present study. For these measurements, it is critical that the wire is only loaded elastically. Elastic loading can be identified by the symmetry of the loading/unloading response curves.

To extract mechanical properties, the loading curves in Figure 1a are fit to the model in Eq. (1). An example of a loading curve fit can be seen in Figure $1 \mathrm{~b}$ and it is immediately evident that the mechanical response of this wire is not well described by the above-described zeroresidual stress model, Eq. (1). Moreover, the $F-d$ response is strongly linear with the cubic dependence on displacement, $\Delta z_{\text {center, }}$ being less than that predicted by the model. The measured displacement $(100 \mathrm{~nm})$ is greater than 3 times the radius of the wire $(32 \mathrm{~nm})$ and should be well in the cubic stretching regime according to Eq. (1); see supplemental Figure S1. The increased linear response is consistent with the wire being subjected to a residual tensile stress. This residual tensile stress results in enhanced stiffening of the beam. From Figure $1 \mathrm{~b}$ it can be observed that a larger force is required to push the wire by a given displacement when compared to that predicted by Eq. (1).

Intuitively, one possible cause for this residual stress is the mechanical clamping process. This can be investigated directly for those wires where adhesion to the substrate surface is sufficient to pin a nanowire in place. This is the case for the AgNWs used in these measurements. The mechanical clamps do provide additional mechanical stability and they also clearly define the pinning length, L. Without clamps the pinning length is not well defined. Even so, it is important to emphasise that the chosen pinning length only affects the magnitude of Young's modulus obtained from the fit (see supplemental Figure S2) it does not affect the overall fit of the model. The inset of Figure 1d shows an AFM image of a $64 \mathrm{~nm}$ diameter AgNW suspended 
over a trench without Pt mechanical clamps. The $F-d$ response of this nanowire is shown in Figure 1c along with the subsequent fit to the doubly-clamped beam model in Figure 1d. From the repeatable $F-d$ response it is clear that the wire is pinned to the substrate. However, based on the poor fit it is evident that the wire is still subjected to a residual axial stress (tension).

The observed residual stress may be a result of the solvent process for depositing nanowires. This type of residual stress has been observed before by Hudson et al. ${ }^{19}$ where the Heidelberg model $^{12}$ was adapted to describe spider silk that was subjected to an initial residual stress. ${ }^{19}$ The modification to the Heidelberg model involves adding a pretension term $\alpha_{0}$ to account for this residual stress. The $f(\alpha)$ term is modified so that; ${ }^{20}$

$$
\alpha=\alpha_{0}+\frac{6 \epsilon(140+\epsilon)}{350+3 \epsilon}
$$

where

$$
\alpha_{0}=\frac{T L^{2}}{E I}
$$

which represents the ratio of residual axial stress to the stress generated by bending. $T$ is the residual tension in the wire. The residual stress can occur in the form of tension (positive $\alpha_{0}$ ) or compression (negative $\alpha_{0}$ ). The additional $\alpha_{0}$ term in Eq. (4a) affects the $F-d$ response of the beam in the small (linear) displacement range, as described previously. When a beam exhibits a residual axial stress, the first term in Eq. (4a), that describes the residual stress, is fit to our measured data, we observe excellent agreement, see Figure 1d. However, the extracted Young's modulus of $34 \mathrm{GPa}$ from this fit is much lower than the expected bulk value of 83 GPa for silver. The likely reason for this anomaly is that the Young's modulus, $E$, and tension, $T$, both affect the linear response of the $F$ - $d$ curve at small displacements, $\Delta z_{\text {center. }}$ As such, their effects cannot be de-coupled easily in the absence of data at large displacements (where the cubic term dominates). True cubic behaviour of the $F-d$ curve is rarely observed for the metal wires studied here. Indeed for many such wires, including semiconductor wires, large 
displacements may result in failure or plastic deformation. ${ }^{12}$ This inability to separate the effects of Young's modulus and residual tension is found in many other wires that we studied (data not shown), and presents a significant bottleneck to the characterisation of these wires in the presence of residual tension.

Therefore, it is clear that a different approach is required to (i) demonstrate the presence of residual stress and (ii) to unequivocally measure the Young's modulus of the wire. We start with the unclamped beam in Figure $2 \mathrm{a}$ and adopt an approach described by Wong et al. ${ }^{16}$ Namely, we section the AgNW into two single-clamped cantilevers. Since each wire section is pinned at one end only ensures that they cannot be subjected to an axial residual stress - any potential axial stress is released at the free end. This measurement differs in important ways from the doubly-clamped beam method. Rather than loading the nanowire at the same centrepoint position, the NW is loaded at multiple positions along its length as depicted in Figure $2 \mathrm{~d}$. In Euler-Bernoulli beam mechanics, the deflection, $d$, at the free end of a cantilever is, ${ }^{21}$

$$
d=\frac{F L^{3}}{3 E I}
$$

Rearranging this equation, gives

$$
F^{-\frac{1}{3}}=(3 E I d)^{-\frac{1}{3}} \cdot\left(L_{c}+\Delta L\right)
$$

where $L_{c}+\Delta L$ is the length of the NW from its pinned end to its free end; $L_{c}$ is the nominal wire length from its pinned end to the load point, and $\Delta L$ is the required adjustment to give the true length. The latter length, $\Delta L$, is fixed for any given wire because wire pinning is not adjusted as the load point is varied. This formula can be used to assess the accuracy of the length, $L$, used in the doubly-clamped beam experiments. The wire is loaded and unloaded so as to test for and avoid plastic deformation. The $F-d$ response as the nanowire is loaded at different positions along its length is shown in Figure 3a. The initial $F-d$ response is linear in accordance with Eq. (5). As the wire is loaded closer to its pinning point the force experienced 
by the tip increases. For each of the $F-d$ curves the slope of the linear section of the loading curve is obtained, as shown in Figure 3b. At larger displacements, the slopes become nonlinear, presumably as the tip starts to slip over the wire length. For this reason, these non-linear sections of the $F$ - $d$ curve are not used in the analysis. From the instantaneous slope at any given displacement, $d$, the force can be calculated according to Eq. (6) and a plot of $F^{-\frac{1}{3}}$ vs $L_{c}$ generated, see Figure 3c. The Young's modulus can be extracted from the slope of this plot. An important advantage of this technique is that the exact pinning point of the nanowire is not required - Young's modulus determination depends only on the slope of the resulting straight line. Even so, the intercept of this line with the x-axis allows the true pinning length to be determined, $L_{c}+\Delta L$. This represents a significant advantage over the doubly-clamped beam method where an uncertain pinning length can lead to large uncertainty in the extracted Young's modulus.

For the $64 \mathrm{~nm}$ AgNW in Figure 3 the Young's modulus obtained from the doubly-clamped beam experiment is $64 \mathrm{GPa}$, using the (zero-residual stress) Heidelberg model, Eq. (1), ${ }^{12}$ is close to the bulk value of $83 \mathrm{GPa}$ - but, fit of the model to the measured $F$ - $d$ curve is poor. In contrast, the Hudson model, Eq. (4a), ${ }^{19}$ yields an excellent fit but an unreasonably low Young's modulus of $34 \mathrm{GPa}$. When the wire is sectioned using FIB and the measured $F-d$ curve is analysed using Eq. (6), the fit is excellent and the Young's modulus is found to be $75 \mathrm{GPa}$. From the plot intercept in Figure 3c it was determined that the pinning length is $L_{c}+\Delta L=$ $1362 \mathrm{~nm}$. The length, $L_{c}$, from the edge of the trench to the unpinned end of the cantilever wire is $1100 \mathrm{~nm}$. The difference between the two values indicates that the wire is pinned a distance $262 \mathrm{~nm}$ greater than the edge of the trench - this only accounts for one side of the doublyclamped beam, from the cantilevered wire measurement. There is also inaccuracy in the estimated length of the doubly-clamped beam on the other side of the wire. 
Even so, this comparison between the sectioned wire (cantilever) and doubly-clamped wire measurements demonstrates that significant uncertainty can result in the estimation of the true pinned length of the wire. This indicates that for doubly-clamped wire measurements, it is critical that the pinning position be well-defined - this is achieved by depositing Pt clamps on the wire at the edge of the trench, so that the trench width can be used as the true pinning length. It should be emphasised that errors in the pinning length, $L$, used in Eq. (1) only affects the magnitude of the modulus extracted - it cannot affect the quality of fit to the model. Results for 16 other cantilever experiments on AgNWs, are given in Figure 4. The average Young's modulus obtained is $96 \pm 20 \mathrm{GPa}$, which compares well to the bulk value of $83 \mathrm{GPa}$ and other measurements made on silver nanowires. ${ }^{17,22,23}$

Finally, we compare all three approaches: doubly-clamped beams that are fit to (i) the Heidelberg zero-residual stress model in Eq. (1), ${ }^{12}$ and (ii) the Hudson model ${ }^{19}$ that includes the effects of residual stress in Eq. (4a), and separately the sectioned cantilever beam measurements that are fit to Eq. (6). We use silicon nanowires (SiNWs) for this purpose. Previously, we showed that $F-d$ curves of SiNWs are well described by the Heidelberg model and hence the effects of residual axial stress are expected to be small. ${ }^{12}$ The Hudson model ${ }^{19}$ reverts to the Heidelberg model ${ }^{12}$ in cases of zero residual tension, i.e., $\alpha_{0}=0$. Results for a single SiNW measurement analysed in this way are given in Figure 5. From Figure 5a we observe an excellent fit of the Heidelberg model, showing that the SiNW is indeed not affected by residual stress. A Young's modulus of $133 \pm 10 \mathrm{GPa}$ is obtained. The nanowire is then FIB sectioned and analysed using the cantilever beam experiment. The Young's modulus obtained is $128 \pm 13 \mathrm{GPa}$, confirming that both methods yield identical results which are in close agreement with the expected bulk value of $169 \mathrm{GPa}$ and that measured for SiNWs. ${ }^{24,25}$ From Figure $5 \mathrm{c}$ it is clear that the Hudson model $^{19}$, that incorporates residual axial stress, also provides an excellent fit to measurements. However, large variations in the extracted Young's 
modulus are obtained from the model as the displacement fit range is varied, see Figure $5 \mathrm{~d}$. The Heidelberg model gives significantly more consistent Young's moduli as a function of fit range. Another example can be found in the supplemental information (Fig S3). This highlights the challenges in interpreting measurements using a model that includes the effects of residual stress - due to equivalent contributions of Young's modulus and residual tension to the small displacement linear region of the $F-d$ curve; see above. These results strongly suggest that doubly-clamped nanowires should be fabricated, whenever possible, to exhibit minimal residual axial stress and the Heidelberg model, Eq. (1), used to interpret their $F$ - $d$ measurements.

To conclude we have shown that it is possible for nanowires that are fabricated in a doublyclamped beam configuration to exhibit a residual axial stress. Measurement of the $F-d$ curve of such wires and its interpretation using a theoretical model that incorporates the effects of residual tension was found to be problematic - a strong dependence of the extracted Young's modulus on displacement fit range was observed. Sectioning of the nanowires using a focused ion beam and conducting cantilever beam measurements enabled unequivocal extraction of the Young's modulus in the absence of residual stress. This allowed robustness of (i) the zeroresidual stress model of Heidelberg et al., ${ }^{12}$ and (ii) the model of Hudson et al. ${ }^{19}$ which includes residual stress, to be assessed. Our study suggests that, given the limited displacement range possible in metal and semiconductor nanowire measurements, material characterisation should only be performed for doubly-clamped nanowires that do not exhibit residual stress. Use of a model that incorporates residual stress can lead to ambiguity in the measurement of Young's modulus as a function of displacement.

Supporting Information Available: Supplementary information accompanies this paper. This material is free of charge via the Internet at http://pubs.acs.org. 
Figures
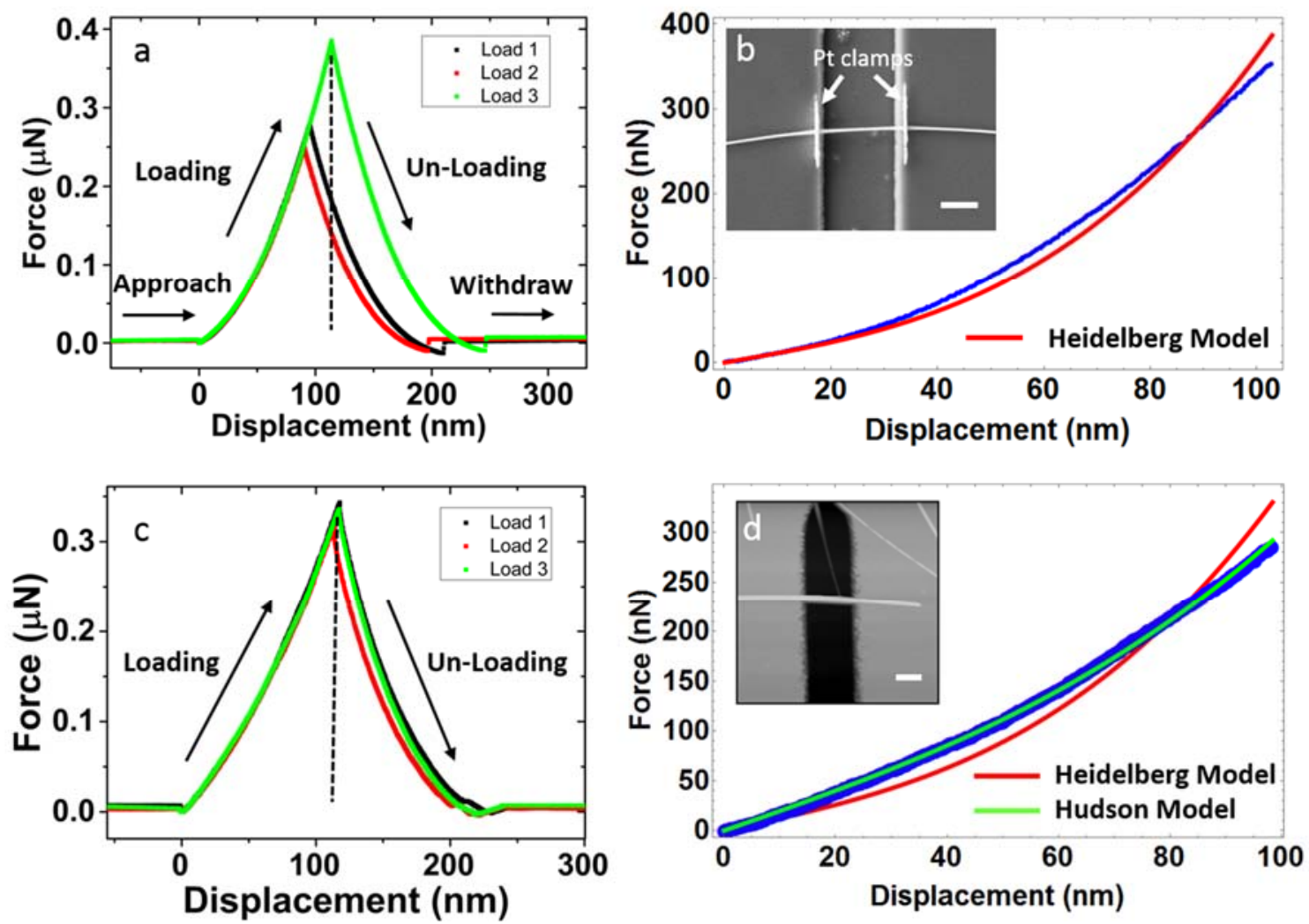

Figure 1. (a) Repeatable $F-d$ curves for a $64 \mathrm{~nm}$ diameter $\mathrm{AgNW}$ deposited over a trench and clamped using Pt-EBID mechanical clamps. (b) Loading curve 3 from (a) fitted to the Heidelberg model described by Eq. (1). Inset shows a AgNW clamped over a trench using PtEBID clamps. (c) Repeatable $F-d$ curves for a $64 \mathrm{~nm}$ diameter AgNW clamped over a trench by surface adhesion. (d) Loading curve 3 from (c) fitted to both the Heidelberg model (Red) and Hudson model (Green). Inset shows a AgNW deposited over a trench and adhered by surface adhesion only. All scale bars, $1 \mu \mathrm{m}$. 


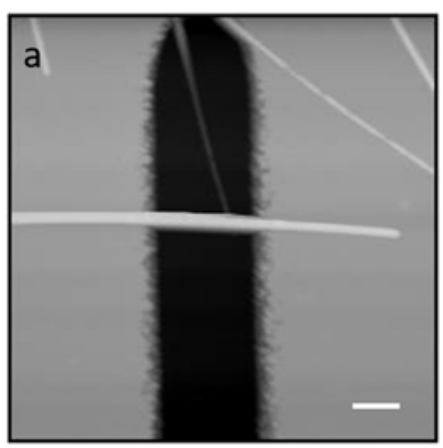

b
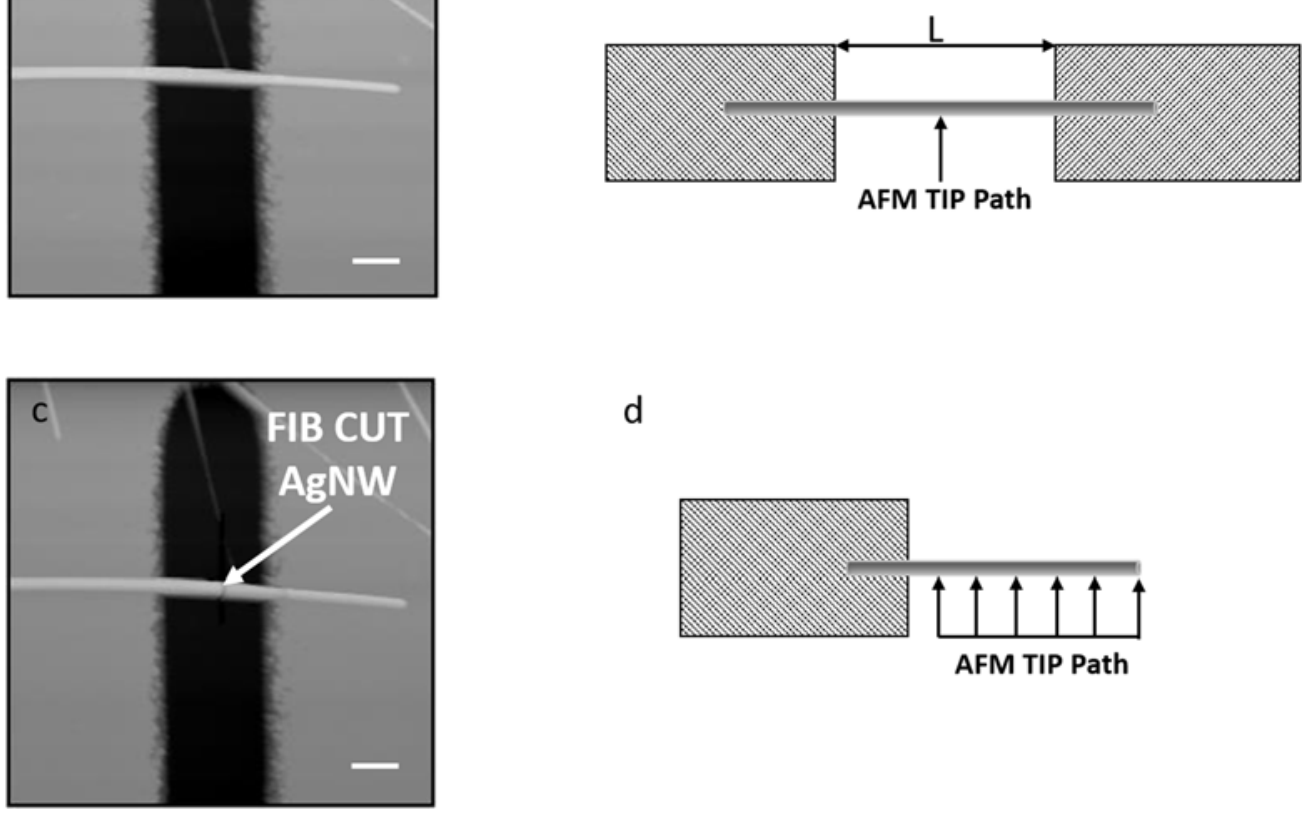

d

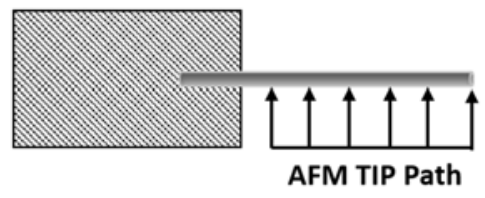

Figure 2. (a) AFM image of a $\mathrm{AgNW}$ suspended over a trench for the 3-point bending experiment. (b) Schematic of 3-point bending experiment. (c) AFM image of the same AgNW after being sectioned with a focused ion beam. (d) Schematic of cantilever experiment on single-clamped AgNW. All scale bars, $1 \mu \mathrm{m}$. 

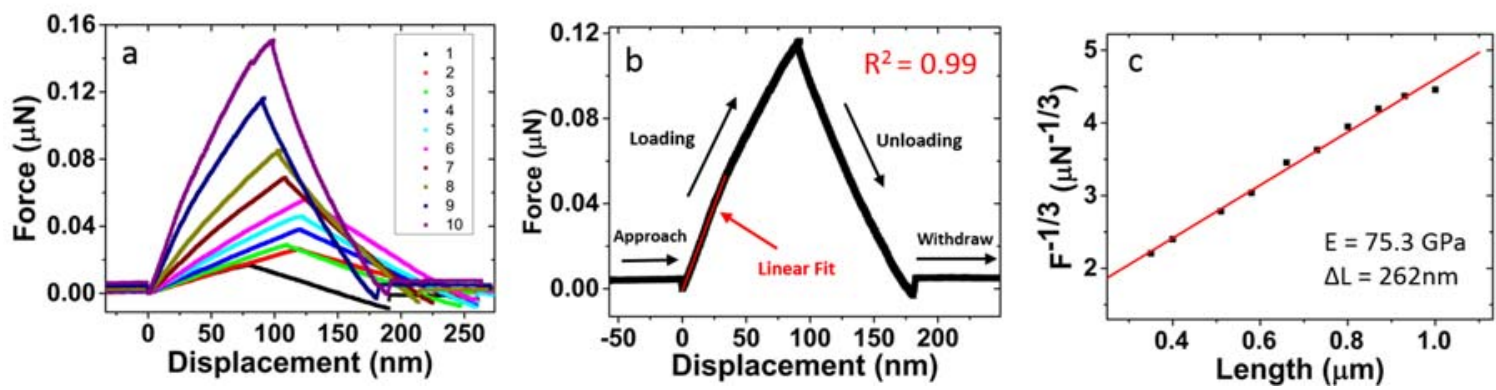

Figure 3. (a) $F-d$ response of a single-clamped AgNW loaded/unloaded at different positions along its length. (b) Individual loading/unloading curve from (a) showing the linear region used for analysis. (c) Plot of $\mathrm{F}^{-1 / 3}$ vs L from which Young's modulus can be deduced from the slope and the inaccuracies in the assumed pinning length can be extracted from the intercept. 


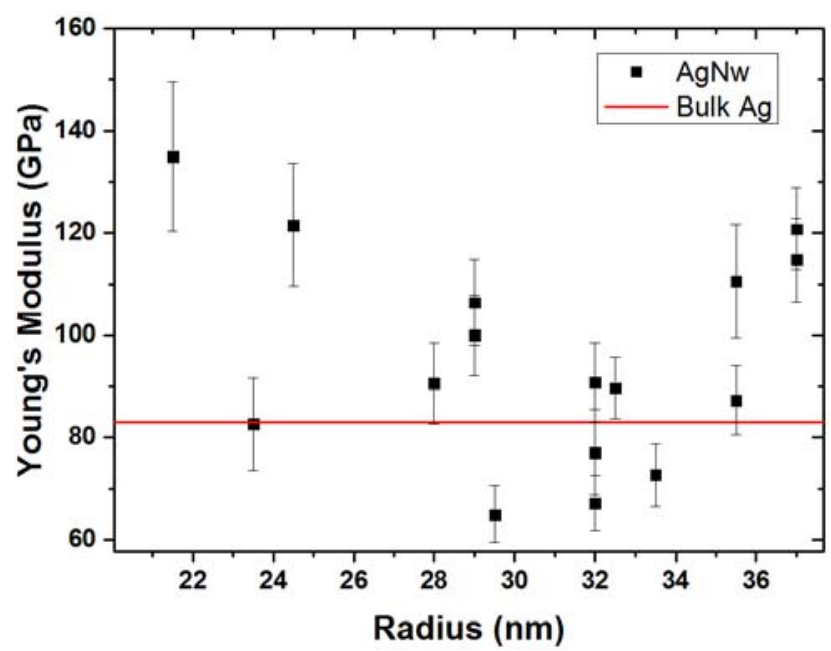

Figure 4. Young's modulus of AgNWs measured using the cantilever beam experiment. 

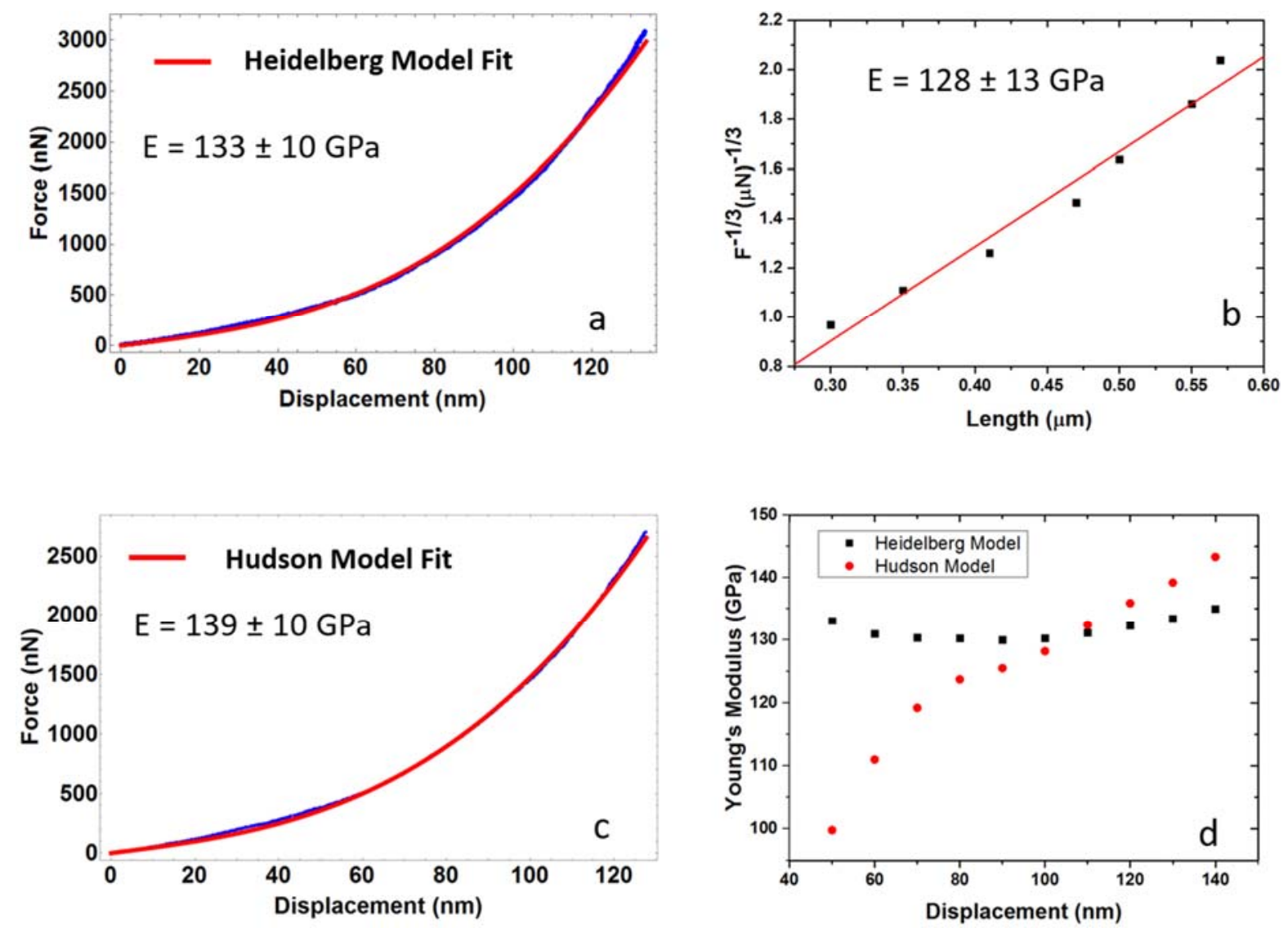

Figure 5. SiNW not affected by initial residual stress analysed using doubly-clamped beam and cantilever beam methods and fit to different models; (a) Heidelberg model, (b) Cantilever beam model, (c) Hudson model, (d) Variation of Young's modulus for Heidelberg and Hudson model when fit over different displacement ranges. 


\section{AUTHOR INFORMATION}

\section{Corresponding Author}

*Email: jboland@tcd.ie

\section{Author Contributions}

S.M. wrote the manuscript, designed and carried out the experiments. J.E.S. developed the mathematical models used to describe the data. J.J.B. wrote the manuscript and led the overall effort. S.M., J.E.S., and J.J.B. discussed the results and contributed to the writing of the manuscript.

\section{ACKNOWLEDGEMENT}

The authors would like to thank Prof. James F. Cahoon and David J. Hill at the University of North Carolina, Chapel Hill, North Carolina for supplying us with the silicon nanowires used in this work and the staff at the Advanced Microscopy Lab (AML), CRANN, Trinity College Dublin. This publication has emanated from research supported in part by a research grant from Science Foundation Ireland (12/IA/1482), support from the European Research Council under Advanced Grant 321160 and through the generous support of the Naughton Foundation. 


\section{REFERENCES}

(1) Wu, B.; Heidelberg, A.; Boland, J. J. Nat. Mater. 2005, 4 (7), 525-529.

(2) Wen, B.; Sader, J. E.; Boland, J. J. Phys. Rev. Lett. 2008, 101 (17), 1-4.

(3) Liang, H.; Upmanyu, M.; Huang, H. Phys. Rev. B - Condens. Matter Mater. Phys. 2005, 71 (24), 1-4.

(4) Chen, C. Q.; Shi, Y.; Zhang, Y. S.; Zhu, J.; Yan, Y. J. Phys. Rev. Lett. 2006, 96 (7), 14.

(5) Poncharal, P.; Wang, Z. L.; Ugarte, D.; de Heer, W. A. Science (80-. ). 1999, 283 (5407), 1513-1516.

(6) Chen, X.; Zhang, S.; Wagner, G. J.; Ding, W.; Ruoff, R. S. J. Appl. Phys. 2004, 95 (9), 4823-4828.

(7) Zhu, Y.; Espinosa, H. D. Proc. Natl. Acad. Sci. U. S. A. 2005, 102 (41), 14503-14508.

(8) Yu, M.-F.; Files, B. S.; Arepalli, S.; Ruoff, R. S. Phys. Rev. Lett. 2000, 84 (24), 55525555.

(9) Li, X.; Gao, H.; Murphy, C. J.; Caswell, K. K. Nano Lett. 2003, 3 (11), 1495-1498.

(10) Ni, H.; Li, X. Nanotechnology 2006, 17 (14), 3591-3597.

(11) Paulo, a. S.; Bokor, J.; Howe, R. T.; He, R.; Yang, P.; Gao, D.; Carraro, C.; Maboudian, R. Appl. Phys. Lett. 2005, 87 (5), 2005-2007.

(12) Heidelberg, A.; Ngo, L. T.; Wu, B.; Phillips, M. A.; Sharma, S.; Kamins, T. I.; Sader, J. E.; Boland, J. J. Nano Lett. 2006, 6 (6), 1101-1106.

(13) Almecija, D.; Blond, D.; Sader, J. E.; Coleman, J. N.; Boland, J. J. Carbon N. Y. 2009, 47 (9), 2253-2258.

(14) McCarthy, E. K.; Bellew, A. T.; Sader, J. E.; Boland, J. J. Nat. Commun. 2014, 5 (5), $1-7$.

(15) Salvetat, J.-P.; Briggs, G.; Bonard, J.-M.; Bacsa, R.; Kulik, A.; Stöckli, T.; Burnham, N.; Forró, L. Phys. Rev. Lett. 1999, 82 (5), 944-947.

(16) Wong, E. W.; Sheehan, P. E.; Lieber, C. M. Science (80-. ). 1997, 277 (5334), 19711975.

(17) Wu, B.; Heidelberg, A.; Boland, J. J.; Sader, J. E. Nano Lett. 2006, 6 (3), 468-472.

(18) Ngo, L. T.; Almecija, D.; Sader, J. E.; Daly, B.; Petkov, N.; Holmes, J. D.; Erts, D.; Boland, J. J. Nano Lett 2006, 6 (12), 2964-2968.

(19) Hudson, S. D.; Zhurov, V.; Grbić, V.; Grbić, M.; Hutter, J. L. J. Appl. Phys. 2013, 113 (15), 1-7.

(20) Yaish, Y. E.; Calahorra, Y.; Shtempluck, O.; Kotchetkov, V. J. Appl. Phys. 2015, 117 (16), 1-11.

(21) Beer, F. P.; Johnston, E. R.; DeWolf, J. T.; Mazurek, D. F. Mechanics of Materials (Sixth Edition), 6th Editio.; McGraw-Hill: New York, 2012.

(22) Cuenot, S.; Frétigny, C.; Demoustier-Champagne, S.; Nysten, B. Phys. Rev. B 2004, 69 (16), 1-5.

(23) Zhu, Y.; Qin, Q.; Xu, F.; Fan, F.; Ding, Y.; Zhang, T.; Wiley, B. J.; Wang, Z. L. Phys. Rev. B - Condens. Matter Mater. Phys. 2012, 85 (4), 1-7.

(24) Zhu, Y.; Xu, F.; Qin, Q.; Fung, W. Y.; Lu, W. Nano Lett. 2009, 9 (11), 3934-3939.

(25) Sohn, Y.-S.; Park, J.; Yoon, G.; Song, J.; Jee, S.-W.; Lee, J.-H.; Na, S.; Kwon, T.; Eom, K. 2009, 5 (1), 211-216. 


\title{
Supplemental Information
}

\section{Material Characterisation of Nanowires with Intrinsic Stress.}

\author{
Shaun Mills ${ }^{\dagger \S}$, John E. Sader ${ }^{\perp}$, John J. Boland ${ }^{\dagger \S}$ \\ ${ }^{\dagger}$ School of Chemistry, Trinity College Dublin, Dublin 2, Ireland \\ $\S$ Centre for Research on Adaptive Nanostructures and Nanodevices (CRANN), Trinity \\ College Dublin, Dublin 2, Ireland \\ ${ }^{\perp}$ School of Mathematics and Statistics, University of Melbourne, Victoria 3010, Australia
} KEYWORDS

Residual stress, doubly-clamped, nanowires, Young's modulus, Euler-Bernoulli 


\section{Non-Linear F-d Response of a Doubly-Clamped Beam}

When a nanowire, in a doubly-clamped beam configuration is loaded at its centrepoint, initially a linear F- $d$ response is observed which accounts for elastic beam bending. However, at displacments greater than the radius of the wire the response becomes non-linear. An asymptotic cubic response at large displacements results due to tensile stresses induced along the length of the wire by stretching. Figure S1 shows simulated F-d curves for a nanowire of diameter $64 \mathrm{~nm}$ with a pinning length, $L$, of $2.2 \mu \mathrm{m}$ at increasing displacements as a function of the radius.

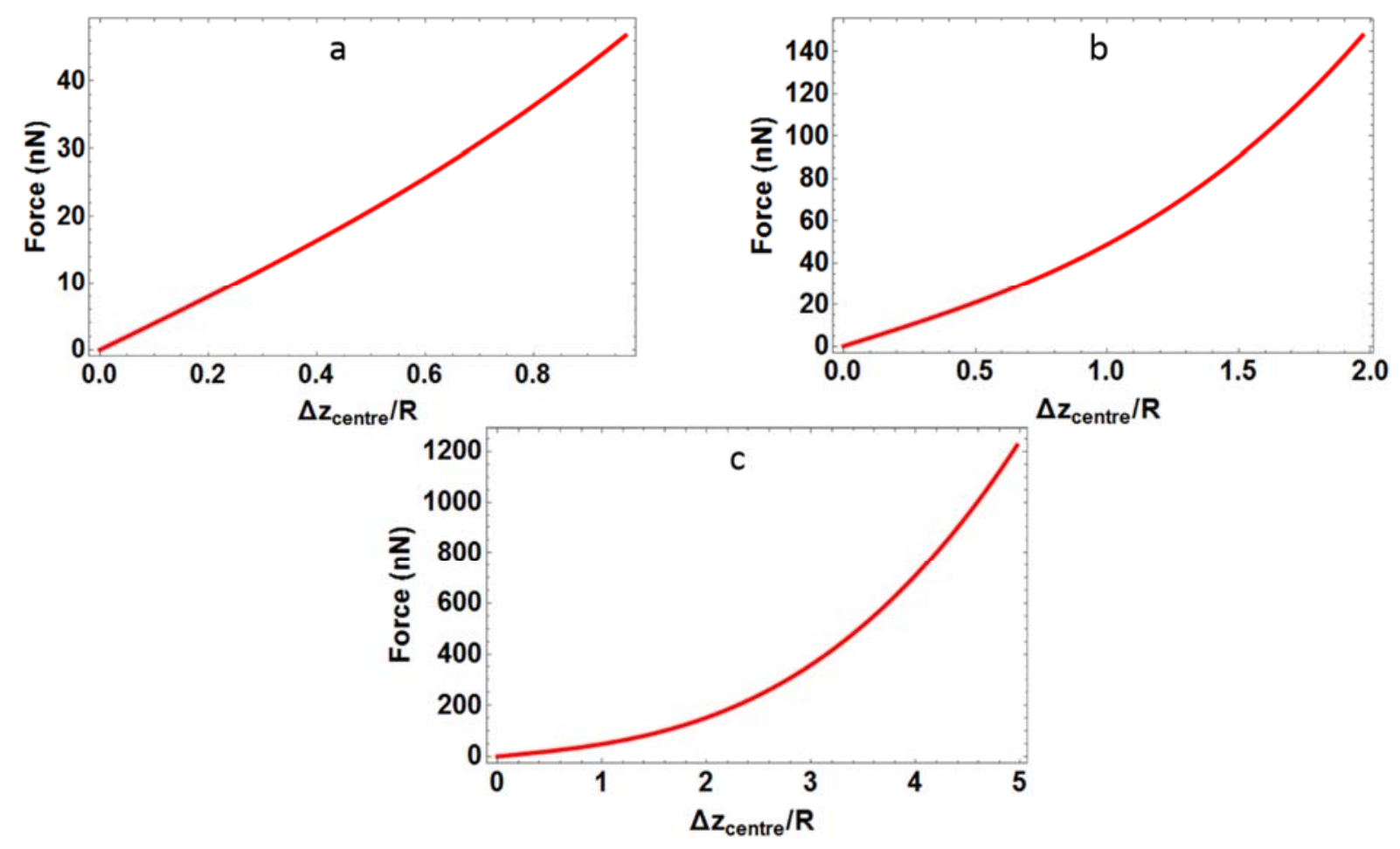

Figure S1. Simulated $F$ - $d$ curves for a circular cylinder showing the non-linearity for a maximum (a) one radius. (b) 2 radii. (c) 5 radii. 


\section{Effect of Incorrect Pinning Length on Heidelberg Model Fit and Young's}

\section{Modulus Extraction}

To correctly extract the mechanical properties from the Heidelberg model fit to the F-d response, certain parameters are required. One is the pinning length, $L$, of the NWs, which is normally defined using mechanical clamps generally deposited using EBID of a metal such as platinum. To verify whether the mechanical clamps are straining the NWs, they are not deposited. The AgNWs in these experiments are adhered to the surface by surface adhesion, however there is a greater uncertainty in the pinning length when no physical mechanical clamps are deposited. Figure S2 shows that when using different pinning lengths for the determination of Young's modulus, it only affects the magnitude of Young's modulus extracted. It does not affect the overall fit to the model so the use of an incorrect pinning length does not affect the residual stress apparent in the NW under investigation.
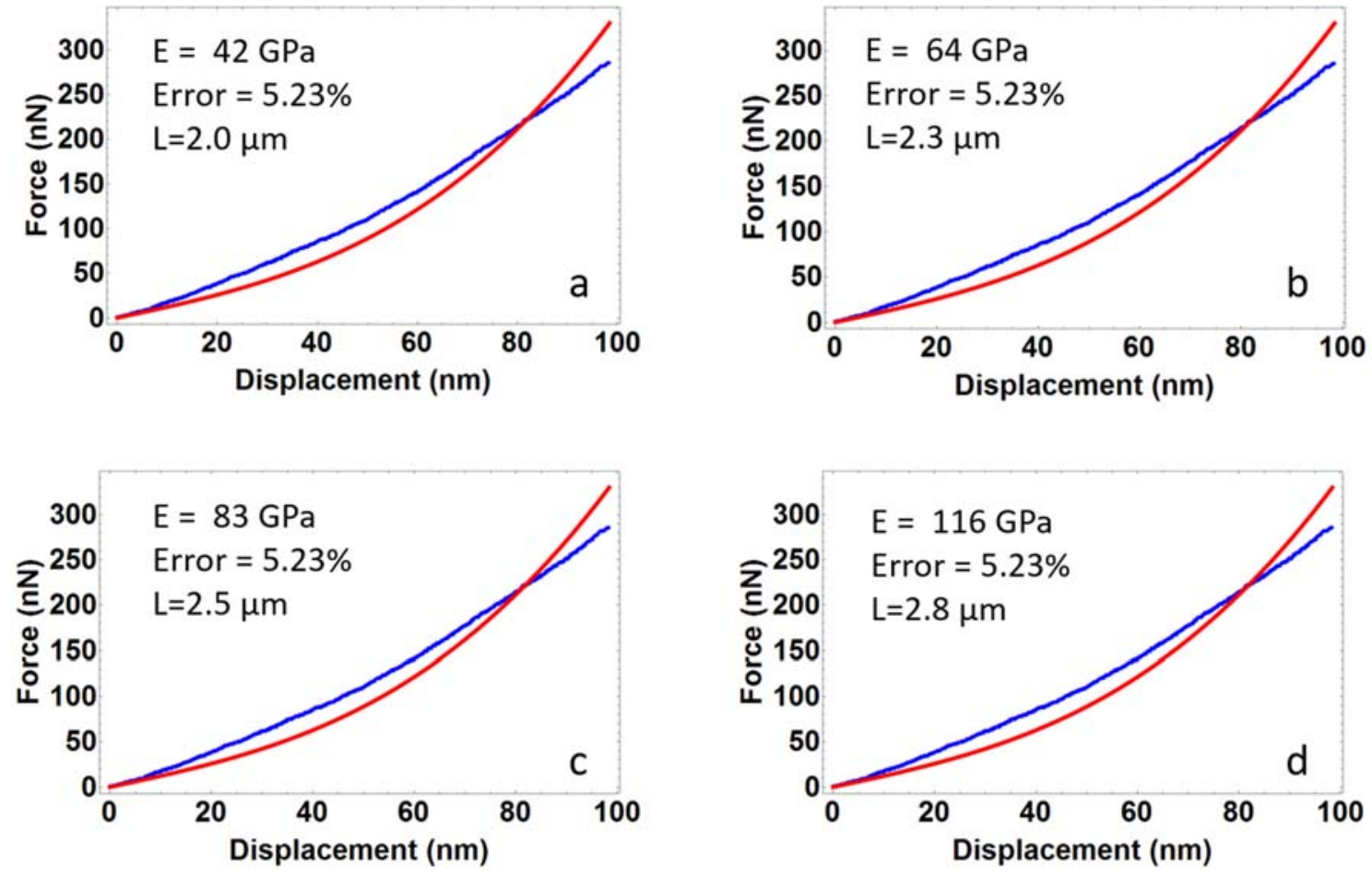

Figure S2. Quality of the fit plus extracted Young's modulus when the experimental data is

fit to the Heidelberg model at different pinning lengths; (a) $2 \mu \mathrm{m}$, (b) $2.3 \mu \mathrm{m}$, (c) $2.5 \mu \mathrm{m}$, (d) $2.8 \mu \mathrm{m}$. 


\section{Comparison of Doubly-Clamped and Cantilever Beam Methods}

Another example of a SiNW which is not subjected to an initial residual stress can be seen in Figure S3. The Young's modulus of this wire is first obtained using the doubly-clamped beam method and Heidelberg model which gives a Young's modulus of $161 \pm 11 \mathrm{GPa}$. The wire is subsequently sectioned using FIB and analysed using the cantilever beam experiment. This gives a Young's modulus of $172 \pm 13 \mathrm{GPa}$, again validating the doubly-clamped beam method and Heidelberg model for wires under no residual stress. Again when the data is fitted to the Hudson model the inconsistency in the modulus over a wide range is observed.
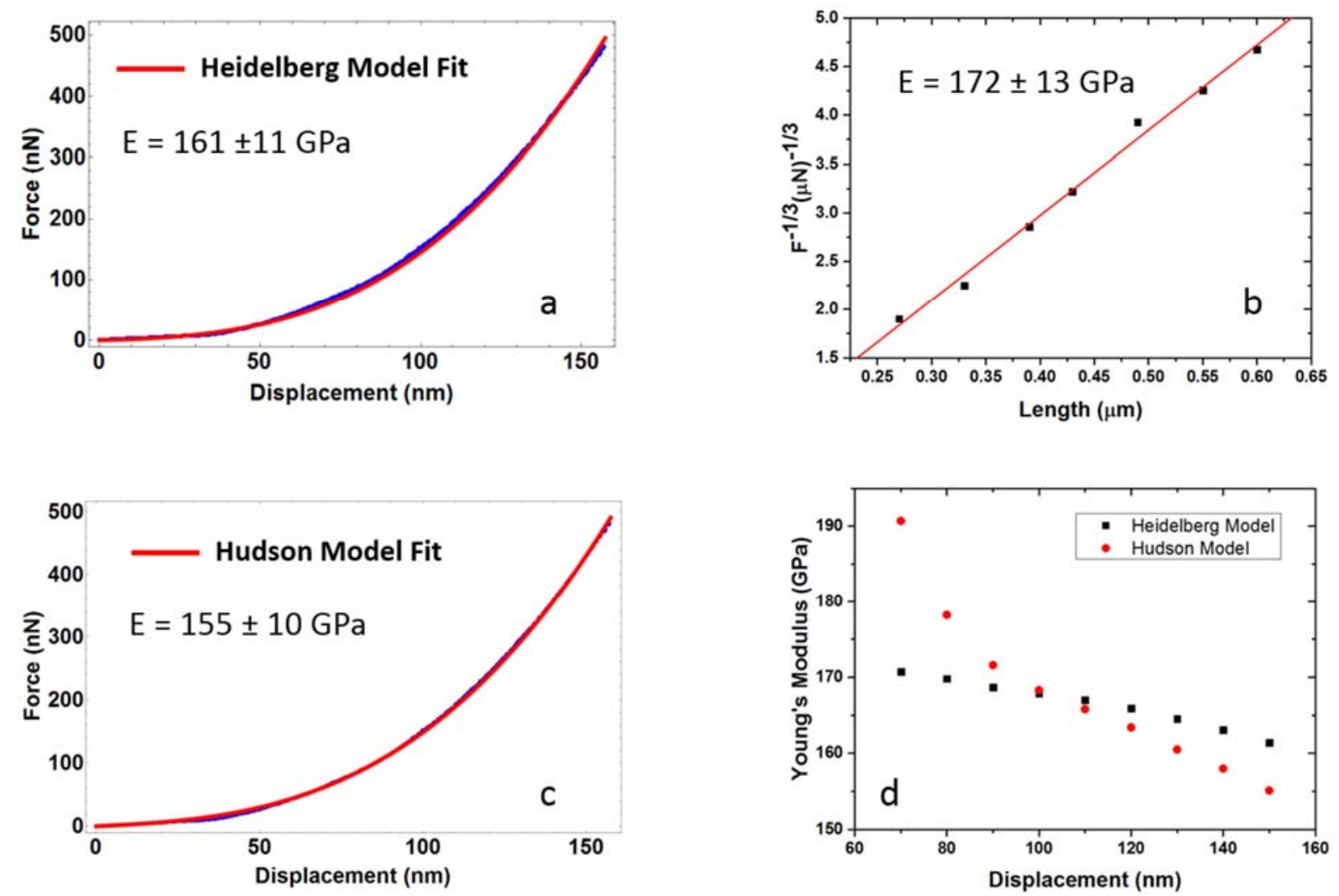

Figure S3. SiNW not affected by initial residual stress analysed using doubly-clamped and cantilever beam methods and fit to different models. (a) Heidelberg model, (b) Cantilever beam model, (c) Hudson model, (d) Variation of Young's modulus for Heidelberg and Hudson model when fitted over different displacement ranges. 